Design Cohort study.

Setting Women's Hospital.

Subjects and methods A representative sample of 1,608 women expressed their consent to study. Questionnaire covered variables related to socio-demographic factors, family history, medical history, maternal complications and neonatal outcome.

Results The prevalence of GDM in Qatar was 16.3\%. Women with GDM were significantly higher in the age group of 35-45 years $(45 \% ; \mathrm{p}=0.001)$. Family history of DM $(31.7 \%$; $\mathrm{p}<0.001)$, increased parity $(55.3 \%$; $\mathrm{p}=0.004)$ and obesity $(59.2 \%$; $\mathrm{p}<0.001)$ were determinants of GDM in pregnant women. Maternal complications like pregnancy induced hypertension ( $19.1 \%$ vs $10.3 \%$; $p<0.001$ ), preclampsia $(7.3 \%$ vs $3.8 \% \mathrm{p}=0.012)$, antepartum hemorrhage $(19.2 \%$ vs $14.6 \%$; $p=0.05)$ and Caesarean $(27.9 \%$ vs $12.4 \%$; $p<0.001)$ were significantly higher in GDM women. Neonates were at increased risk of preterm birth $(12.6 \%$ vs $8.3 \%$; $=0.03)$, macrosomia $(10.3 \%$ vs $5.9 \% ; \mathrm{p}=0.01$ ) and birth trauma ( $8 \%$ vs $3 \% ; \mathrm{p}<0.001)$. Advanced age group $(p=0.001)$, obesity $(P<0.001)$, Family history of $D M$ $(\mathrm{P}<0.001)$ Macrosomia $(\mathrm{p}=0.05)$, Antepartum hemorrhage $(\mathrm{p}=0.001)$, Caesarean $(p<0.001)$ were the significant associated factors for GDM.

Conclusion The GD was higher in women and they were at increased risk of developing maternal and neonatal complications. The advanced maternal age, family history of diabetes, macrosomia, obesity and caesarean delivery were the main significant associated risk factors for GDM.

\section{CLINICAL AND EPIDEMIOLOGIC CHARACTERISTICS OF TYPE 1 DIABETES IN CHILDREN IN A PEDIATRIC UNIT FROM SFAX (TUNISIA)}

doi:10.1136/archdischild-2012-302724.0641

H Aloulou, S Kmiha, I Maaloul, L Sfaihi, I Chabchoub, T Kammoun, M Hachicha. Pediatric, Hedi Chaker Hospital, Sfax, Tunisia

Introduction During the last few decades, an increase in the incidence of type 1 diabetes (DT1) in children was reported in most parts of the word.

Aims Study the epidemiologic and clinical particularity of (DT1) in our patients.

Patients and methods From 2000 to 2011, children under 15 years with newly diagnosed type 1 diabetes mellitus and drown from department of pediatrics in Sfax were ascertained retrospectively. Cases of neonatal diabetes were excluded. Patients were devised on 3 groups: group 1: less than 5 years (107 cases), group 2: 5-10 years (120 cases), group 3: $10-16$ years (98 cases).

Results The incidence was 27 new cases/year (17-34 cases). There were 166 boys - 159 girls. Median age at diabetes onset was 7 years and 7 months. Twenty three percent of the children had a familial history of diabetes type 1 significantly more frequent in group 1 . Fifty two percent of all cases were diagnosed in the cold season. The age at introduction of cow milk in alimentation was less than 6 months in $54.4 \%$. Cereals were introduced in alimentation at an age less than 3 months in $12.3 \%$ of cases. Ketoacidosis revealed diabetes in $55.7 \%$ of cases, significantly more frequent in group $1(66.3 \%)$, polyuria and polydipsia were more frequent in group $3(98 \%)$ Hypoglycemia was more frequent in group 1

Conclusion Significant advances have been made in the clinical care, epidemiologic studies have an important on-going role to investigate the complex causes.

\section{POTENTIAL PROTECTIVE EFFECTS OF TRIGONELLA FOENUM GRAECUM AND SODIUM ORTHOVANADATE IN HYPERGLYCEMIA-INDUCED ALTERATIONS IN CARDIAC MEMBRANE IN ALLOXAN DIABETIC RATS}

doi:10.1136/archdischild-2012-302724.0642
P Kumar, RK Kale, NZ Baquer. School of Life Sciences, Jawaharlal Nehru University, New Delhi, India

Objectives Oxidative stress in diabetic tissues is accompanied by high level of free radicals and the simultaneously declined antioxidant enzymes status leading to cell membrane damage. In the present study, the effect of sodium orthovanadate (SOV) and Trigonella foenum graecum seed powder administration has been studied on blood glucose and insulin levels, membrane bound ATPases $\left(\mathrm{Na}+\mathrm{K}+\mathrm{ATPase}, \mathrm{Ca}^{2}+\mathrm{ATPase}\right.$ ), antioxidant enzymes (superoxide dismutase, glutathione S-transferases), lipid peroxidation, lipofuscin accumulations and distribution of glucose transporter (GLUT4) in heart of the alloxan induced diabetic rats and to see whether the treatment with SOV and Trigonella is capable of reversing these effects.

Methods Diabetes was induced by administration of alloxan monohydrate $(15 \mathrm{mg} / 100 \mathrm{~g}$ b.wt.) and female rats were treated with $2 \mathrm{IU}$ insulin, $0.6 \mathrm{mg} / \mathrm{ml} \mathrm{SOV}, 5 \%$ Trigonella in the diet and a combination of $0.2 \mathrm{mg} / \mathrm{ml} \mathrm{SOV}$ with $5 \%$ Trigonella separately for 21 days.

Results Diabetic rats showed hyperglycemia with almost four fold high blood glucose levels. Hyperglycemia increases lipid peroxidation and lipofuscin accumulations, causing decreased activities of membrane bound ATPases, antioxidant enzymes and GLUT4 expression with diabetes in the rat heart. Rats treated with combined dose of vanadate and Trigonella had glucose levels comparable to controls, similar results were obtained with the activities of antioxidant enzymes, membrane bound ATPases, lipofuscin, lipid peroxidation and GLUT4 in diabetic rats.

Conclusion Our results showed that lower doses of vanadate $(0.2 \mathrm{mg} / \mathrm{ml})$ could be used in combination with Trigonella to effectively counter diabetic alterations without any toxic side effects.

\section{ELEVATED ACETOACETATE, OXIDATIVE STRESS AND MCP-1 LEVELS IN CORD BLOOD OF INFANTS OF DIABETIC MOTHERS}

doi:10.1136/archdischild-2012-302724.0643

D Kurepa, A Pramanik, L Groome, J Bocchini, S Jain. LSUHSC, Shreveport, LA, USA

Background Infants of diabetic mothers (IDM) are at increased risk for metabolic complications. Type 1 and some type 2 diabetic patients have elevated levels of ketone bodies acetoacetate (AA) and $\beta$-hydroxybutyrate (BHB) in addition to hyperglycemia. The effect of ketonemia on the inflammatory markers in infants of diabetic mothers is unknown.

Objective The aim of this study is to examine how hyperketonemia in diabetic mothers affects markers of inflammation and oxidative stress in their offspring.

Methods Blood was obtained from 23 diabetic mothers and 13 healthy mothers, and their infants' umbilical cords at the delivery. IL-8, MCP-1 and protein carbonyl (protein oxidation) levels were determined by ELISA. U937 human monocyte cell culture was used to examine the effect of $\mathrm{AA}$ and $\mathrm{BHB}$ on secretion of MCP-1.

Results There was a significant increase in the levels of AA in cord blood of diabetic mothers compared with cord blood of healthy mothers. A significant increase in the levels of protein oxidation $(p<0.05)$ and MCP-1 levels $(p<0.05)$ were observed in the cord blood of IDMs. The level of MCP-1 significantly correlated $(r=0.51$, $\mathrm{p}=0.01$ ) with the concentration of AA in the IDM. In further experiments with cultured monocytes treated with exogenous AA (0-4 $\mathrm{mM}$ ), a significant increase in MCP-1 secretion was observed with $\mathrm{AA}$ but not in BHB-treated monocytes.

Conclusion This study suggest that blood levels of AA, oxidative stress and MCP-1 are elevated in IDM, which may contribute to the development of the metabolic complications seen in IDM. 

AFTER INFLUENZA VIRUS INFECTION IN PAEDIATRIC PATIENTS

doi:10.1136/archdischild-2012-302724.0644

10 Genovese, 'S Pulitanò, ${ }^{2} \mathrm{~S}$ Crocco, ${ }^{1} \mathrm{~A}$ Mancino, ${ }^{1} \mathrm{~F}$ Tosi, ${ }^{1} \mathrm{M}$ Piastra. ${ }^{1}$ Paediatric Intensive Care Unit; ${ }^{2}$ Pediatrics, Policlinico Gemelli University Hospital, Roma, Italy

Influenza virus can cause common respiratory tract infections and rarely multiorgan system disorders, resulting in mild infection, severe respiratory disease or systemic disease and complications. CNS dysfunction, an important complication of influenza infection includes IAE, a rapid progressive encephalopathy that usually presents in the early phase of influenza infection. Because of lack of inflammation in the CNS, IAE is always named influenza-associated acute encephalopathy, which includes acute necrotizing encephalopathy (ANE), presenting with fulminant encephalopathy and characteristic brain lesions following viral infection, which pathogenesis is not fully understood, but associated with unfavourable outcome.

We present four new cases of central nervous system dysfunction subsequent to infection with Influenza Virus. All four cases had convincing evidence of preceding Influenza disease with no evidence of viable Influenza Virus in the cerebrospinal fluid. We propose that these cases represent examples of post Influenza central nervous system dysfunction. We also present a review of the literature regarding Influenza neurologic dysfunction and speculate on the underlying pathologic mechanisms.

\section{ACUTE ENCEPHALITIS COMPLICATING RUBELLA: FOUR CASE REPORTS}

doi:10.1136/archdischild-2012-302724.0645

K Lajmi, S Haddad, S Hammami, C Ben Meriem, L Ghedira, MN Gueddiche. Fattouma Bourguiba Hospital, Monastir, Tunisia

Background Rubella encephalitis is a rare complication of rubella with an incidence that has been reported to range from 1/4300 to $1 / 24000$.

Methods We report four pediatric cases of acute encephalitis complicating rubella during an epidemic of rubella between March 2011 and November 2011 to the Fattouma Bourguiba Hospital.

Results Three males and one female were included. The mean age was 9 years ( $7-12$ years), rubella vaccine have been given only for two patients. Macular rash was reported in three cases. The most clinical symptoms were: headache, fever, loss of consciousness and seizures. Serum immunoglobulin (Ig) M antibodies against rubella virus were present in all cases. In all cases cerebrospinal fluid (CSF) analysis reveals lymphocytic pleocytosis, elevated protein levels and normal glucose levels and the presence of immunoglobulin $M$ antibodies against rubella virus. Electroencephalogram (EEG) showed slow wave activity without focal or paroxysmal features in all cases and computed tomographies of the brain (CT) were normal. Mechanical ventilation was needed in 3. Acyclovir was administered intravenously for all patients given the initial concern of herpes simplex encephalitis. Favourable outcomes in all cases were reported.

Conclusion In Tunisia a revision of our vaccination program against rubella is needed. Benefits and the costs of a non-selective vaccination strategy including all children and all women of childbearing age must be weighted to prevent such a severe complication. FEBRILE CONVULSIONS

doi:10.1136/archdischild-2012-302724.0646
F Akter. East Kent University Hospital Trust, East Kent, UK

Background and Aims Febrile convulsions are the most common seizure disorder in childhood, affecting $2-5 \%$ of children between the ages of 3 and 60 months. It is a frightening and anxiety-provoking event for parent and caregivers. The initial management of this condition is often poor in rural setting in third world countries.

Aims To assess the factors affecting the initial management of children with febrile convulsions.

Methods A prospective study interviewing parents of 20 children with febrile convulsions admitted to a hospital in Dhaka, Bangladesh.

Results 19 mothers and 2 fathers of children with febrile convulsions were interviewed. Only 1 parent knew what a febrile convulsion was. $50 \%$ initially managed their children by massaging oil onto the back. $60 \%$ of the children were seen by a local religious healer. $90 \%$ of parents believed their child was possessed by an evil spirit. $50 \%$ thought their children will die. $60 \%$ of the parents had been educated up to only primary school level. $90 \%$ reported their local hospital to be 10 miles away.

Conclusions The education level, religious beliefs and location of nearest hospital significantly affected the way parents initially manage their children with febrile convulsions. It is important in third world countries that media methods such as television, radio and newspapers are used to discuss management of common conditions to avoid inappropriate treatments by parents. Leaflets should also be made available to collect from local shops and family physicians.

\section{CARDIOVASCULAR ABNORMALITIES IN CHILDREN AND ADOLESCENTS WITH TYPE 1 DIABETES MELLITUS}

doi:10.1136/archdischild-2012-302724.0647

'GC Yılmaz, 'P Boran, ${ }^{2} \mathrm{~L}$ Yılmaz, ${ }^{3} \mathrm{MA}$ Astarcioglu, ${ }^{3} \mathrm{E}$ Erturk, 'E Tutar, 'G Tokuc. 'Dr. Lutfi

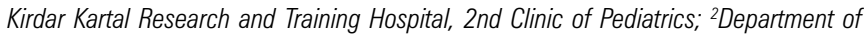
Family Medicine, Dr. Lutfi Kirdar Kartal Research and Training Hospital; ${ }^{3}$ Department of Cardiology, Kosuyolu Heart and Research Hospital, Istanbul, Turkey

Background and Aims Diabetic patients can show signs of cardiac autonomic neuropathy and diabetic cardiomyopathy.

We aimed to determine left ventricular function and cardiac autonomic nervous system function in cardiac-asymptomatic diabetic children and to examine the relationship between cardiac function abnormalities and sex, glycemic control, and diabetes duration.

Methods Left ventricular function was assessed using echocardiography. HRV was computed for each subject by means of standard 24-h time domain measures. The final data analysis was conducted on 57 diabetic patients (mean age $14.4+3.09$ years, 31 males) and 40 healthy controls (mean age $13.6+2.2$ years, 19 males).

Results No significant differences were found between patients and controls with respect to systolic and diastolic blood pressure, however heart rate measured during physical exam at rest was significantly higher in the diabetic patient group. HRV indexes were lower in the patient group but not significant. Left ventricular wall dimensions and systolic function were comparable in both groups. The A wave velocities, deceleration time and IVRT were significantly higher in the diabetic group. Tissue Doppler derived myocardial peak systolic velocity (Sm) of LV and the Tei index were normal reflecting the preserved LV systolic function. The mitral early filling velocities (Em) were significantly reduced in the diabetic group, with a consequently significant lower $\mathrm{E} / \mathrm{A}$ ratio.

Conclusions In conclusion, our diabetic patients had signs of significant left ventricular filling abnormalities. Increased heart rate and lowered HRV indexes although not significant may reflect the early evidence of cardiac autonomic nervous dysfunction. 